\title{
Research on technique for improving the accuracy of converting CAD data using FME based on ArcMap
}

\author{
Rong Liu ${ }^{1}$, Guogang Zhao ${ }^{2}$, Jijun Geng ${ }^{1}$ \\ ${ }^{1}$ Faculty of Geomatics, East China Institute of Technology, Nanchang, 321000, China \\ email:rliu@ecit.edu.cn \\ ${ }^{2}$ Jiangxi Provincial Expressway investment Ltd Group Taihe Management and Maintenance \\ Center,Jian,Jiangxi,343709,China
}

Keywords: FME, ArcMap, DWG data, shape data, Annotation data

\begin{abstract}
This article, firstly, reveals the difference between the text annotation of original CAD Map and the results converted to ArcGIS using Feature Manipulation Engine (FME). Then we propose a strategy, extracting the annotation layer information directly from original map on ArcMap platform and figuring out the conversion errors of annotation information, to correct the errors. The strategy not only guarantees high conversion accuracy, but also improves work efficiency dramatically. Taking topographic map of a city for example, an experiment was carried out. The results show that this strategy could better deal with CAD data, and it provides a feasible scheme for topographic map conversion.
\end{abstract}

\section{Introduction}

GIS and CAD technology is active in the important technology in the field of surveying and mapping, geography, computer. Due to their respective characteristics, widely used and different requirements, the data transformation is applied between the two inevitable problems in the construction of geographic information system [1]. At present most of the city's basic geographic information data is stored in AutoCAD data format, although AutoCAD on graphics processing function is stronger, but on the description and analysis of the spatial data information still need to improve, and GIS in spatial information plays a key role, asked to empty data modeling, analysis and management. If we will have the AutoCAD digital topographic map as a foundation for the GIS database established in the form of digital data sources, make full use of existing digital terrain map, can be more efficient to create urban basic geographic information database, so the CAD data to GIS batch conversion has become a key problem[2]. In this paper, according to the instance, by CAD note ArcMap using improved FME conversion layer, deeply discusses the CAD data into ARCGIS data implementation process.

\section{FME technology theoretical basis}

FME (Feature Manipulation Engine, Spatial data Engine operation) Safe launch of new products, a third-party special tool for data transformation, it has a very powerful features, support nearly hundred kinds of formats of data conversion between each other, such as: Auto CAD DWG/DXF, ESRI Arc/Info to Generate, ESRI Export (E00 Uncompressed), ESRI SDE, ESRI Shape, Intergraph MGE, Intergraph Micro Station Design File, the Map Guide SDL, Mapinfo MIF mids, Mapinfo TAB, Oracle Spatial Cartridge (Relational), Oracle SQL Loader, Small world, SDE2.1 etc. [3]. FME contains the software module operating elements of object, read the module data read from the external data source element, element factory module under the control of the user to merger or division, the data conversion module converts these elements from an expression format to another expression format, it is also possible to hang these elements to the external database, write module will support these elements object in a format output [4]. Spatial data include graphical information and attribute information. Spatial data graphical information is the main content of GIS and DWG data, is the carrier of attribute information; Spatial data attribute information classification and 
coding information of GIS and contains all of the business information, is about the graphical information [5]. Using FME and ArcMap transformation goal is to convert DWG format data to ArcGIS. SHP data. DWG data are stored in points layer, each layer at the time of conversion is strictly divided into dot, line, face, note to conversion, the converted data is stored in the database of ArcGIS corresponding element in the layer [6].

In this paper, first using the data from the FME transform CAD data into ArcGIS with the original CAD figure than found note layer has differences with the original, after using ArcMap directly extract note layer found effect and precision are greatly improved.

\section{DWG data and GIS data comparison analysis}

In the Geodatabase, a DWG file corresponds to a data set of Geodatabase, and in the DWG file with the same geometry information elements are assigned to the same class, including point, multipoint, line, surface and note M elements. But the elements of Geodatabase itself with different geometric information and class have also exists certain difference [7].

Table 1 DWG Shape graphics geometry with Geodatabase data attribute table

\begin{tabular}{cccc}
\hline WG data & $\begin{array}{c}\text { Shape graphics geometry } \\
\text { properties }\end{array}$ & Geodatabase data & $\begin{array}{c}\text { Shape graphics geometry } \\
\text { properties }\end{array}$ \\
\hline note & Point & note & Polygon \\
point & Point & point & Point \\
line & Polyline & line & Polyline \\
surface & Polygon & surface & Polygon \\
\hline
\end{tabular}

Test results can be seen from the table, in the CAD data to GIS data conversion based on Geodatabase, among them the dot, line, face three kinds of spatial data format, the conversion process is relatively simple; In the Geodatabase, CAD note information format for Coverage of the Annotation, its graphics geometry properties as the Point, and the format of the GIS's own note information for Feature Class Annotation, its graphics geometry properties for Polygon; It is because of this difference, so in the note information transformation, you will need to adopt the method of relatively complex to implement [8]. This paper puts forward a new solution on this issue, use ArcMap transform CAD note directly into Shape data.

\section{Application}

\section{(1) Data analysis}

1:10000 topographic map of a town CAD data format(DWG). Convert before, need to check the data graph, can use CASS software for processing, also should delete the pseudo node in the graph data, filtering without property entities to further check the data graph. In addition, also need to check the data graph, according to the actual situation such as the serious scale walls should not be closed, or imported into the ArcGIS will come as a surface treatment, in situations such as this will cover house [9]. After completion of inspection, the inspection data to preview, find out the attribute information contained in each layer. Using FME FME Universal Viewer loaded topographic map, in 211 (general) to housing layer. For example, you can see the layer below Point (Point), Line (Line), Area (surface) and Text (notes). In using FME conversion data is therefore attention should be paid to 211 layers, only transformation, point, line, face, the content of the note. If there are other layer is required according to the actual situation to the corresponding layer.

\section{(2) Data transformation}

Open the FME Workbench find need to transform the data of CAD topographic map, and set the folder to store the converted data. Click OK can automatically set up corresponding relation, select the need to transform the layer. Click OK again can automatically establish a mapping relationship, in 211 (general) to housing, for example, has analyzed this layer is a little bit, in front of the line, 
face, note these content, therefore need to convert these data, can be set in the Gemetry Filter to remove other useless mapping relationship, to complete and then click the start switch can automatically complete the conversion process.

\section{(3) Note data analysis}

Since FME directly convert CAD data notes will be converted to a point, and this point is usually located on CAD diagram note the lower left corner, when in ArcMap note points after the content of the mark and the original has certain deviation, in 831 (height), for example, are shown in figure 1 below (red note for FME conversion, black for the original CAD data).

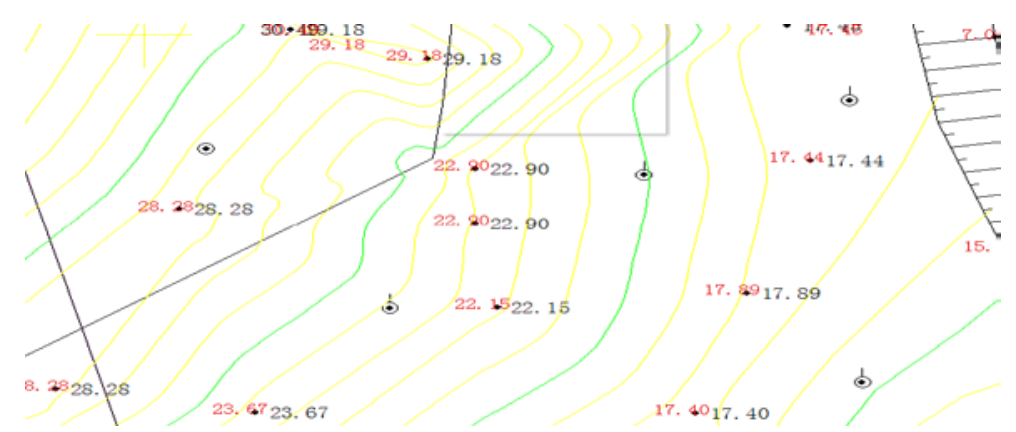

Fig.1. FME conversion notes compared with the original data

The note of FME conversion, data is not lost, but there is a certain deviation on location precision, and every show note need to allocate notes fields, it is very troublesome, or depend on the database, according to the established system can realize but this is not very convenient. ArcMap can realize a method of direct conversion out note content. Using the Import CAD Annotation tool can achieve this goal. Establish a first. GDB database, data is used to store the converted an Annotation, then use ArcMap load to convert CAD data, click on the Import of this CAD Annotation tool, select the original CAD data file and storage. GDB database files, in selecting the appropriate proportion, the conversion can be completed. The converted note data comparison is shown in figure 2 below. As you can see note position to ensure the consistent with CAD note the position of the overlap.

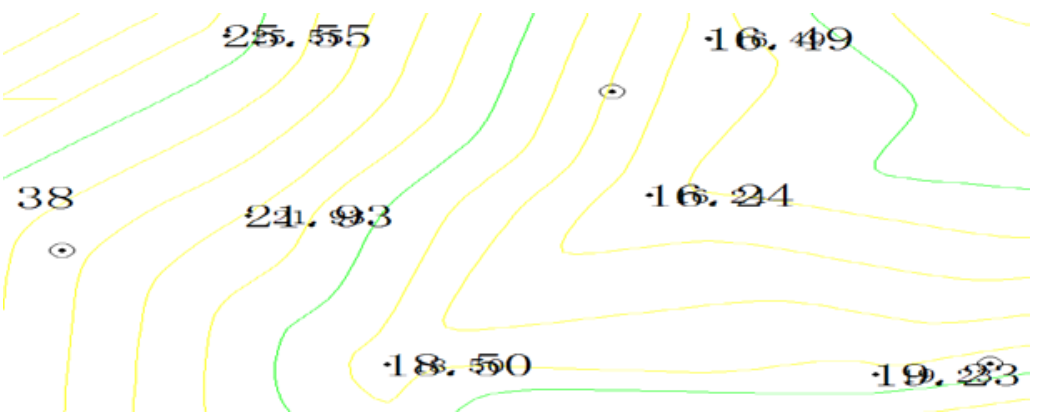

Fig.2. experimental results

\section{Conclusion}

FME in GIS data cross-platform conversion has good versatility and maneuverability, powerful. It has a simple interface, can use different operating mode to achieve the same kind of data in different platforms, or different data on the same platform sharing, various GIS is becoming the DE facto standard for software Shared data [10]. ArcMap flexible application of DWG format note data processing, improve the CAD note data to ARCGIS note data conversion accuracy, proved in this paper, using the method of realizing the quick nondestructive transformation of spatial data, to improve location accuracy note data. Than you can see through the final result, maintain a to data before and after the transformation, to realize the transformation of the data is of great importance, 
for the technical personnel engaged in the development of geographic information system has a certain reference value.

\section{Acknowledgement}

In this paper, the research was sponsored by the Foundation of Key laboratory of watershed ecology and geographical environment monitoring, National Administration of Surveying, Mapping and Geoinformation (Project No. 201512026).

\section{References}

[1] Yunfeng Qiu, Jin Ni. Coordinate conversion between different projection reduction of the [J]. Bulletin of surveying and mapping, 2001, (9): 12-13

[2]Muyao Shi, Auto DWG data with Arc GIS data transformation research [D]. Beijing: China University of Geosciences, 2010

[3]FME technical white paper [Z]. Ann figure Beijing century digital technology development co., LTD, Canada Safe Software Inc FME general agent in China, 2000.

[4]Niansong Chen. FME .Based CAD and GIS data sharing research [D]. Nanjing: nanjing normal university, 2008.

[5]. ESRI ArcMap Tutorial [K]. America: ESRI Press, 2006.

[6]Ying Chen, Yaodong Cheng, Haowen Yan . GIS data by using the FME nondestructive transformation, [J]. Journal of surveying and mapping science, 2007, 32 (2): 75-77

[7]Zhangqi Zhou, Mingjun Peng. Batch conversion method of CAD to the GIS comparison and its implementation based on ArcEngine [J]. Journal of surveying and mapping science, 2010, 35 (5): 161-163.

[8] Xianyong Li. Visual c ++ serial communication technology and engineering practice [M]. Beijing: people's posts and telecommunications publishing house, 2004.

[9]Fan Yang, Chen Bo. Based on the FME DWG data to the study of the application of ArcGIS transformation [J]. Journal of surveying and mapping science, 2012, 5 (2): 143-145

[10] Zhanpai Yao, Ruihong Yuan. FME in the application of GIS data exchange [J]. Journal of guangdong province survey and mapping, 2004, (3) : 27-29. 